\title{
Graphic tools for analysing the influence of noise and aperiodic components in the performance of digital filters: A case study
}

\author{
J. Lázaro ${ }^{1}$, M.A. Zorrozua ${ }^{2}$, J.F. Miñambres ${ }^{2}$, M.I. Sánchez ${ }^{1}$, B. Larrea $^{1}$ and I. Antiza ${ }^{3}$ \\ ${ }^{1}$ Department of Applied Mathematics \\ E.T.S.I., Basque Country University (UPV/EHU) \\ Alda. Urquijo s/n - 48013 Bilbao (Spain) \\ Phone/Fax number:+0034 94601 4150/+0034946014200 , e-mail: jorge.lazaro@ehu.es, \\ ${ }^{2}$ Department of Electrical Engineering \\ E.T.S.I., Basque Country University (UPV/EHU) \\ Alda. Urquijo s/n - 48013 Bilbao (Spain) \\ Phone/Fax number:+0034 94601 4056/+0034 94601 4200, e-mail: miguelangel.zorrozua@ehu.es \\ ${ }^{3}$ GE Multilin \\ Avda. Pinoa 10 - 48170 Zamudio (Spain) \\ Phone number:+0034 94485 8853, e-mail: Inigo.Antiza@IndSys.ge.com
}

\begin{abstract}
An accurate phasor estimation is an important task for a correct operation of control and protection devices. The presence of aperiodic components in electrical signals is a major problem for the correct performance of digital filters. In addition, their stability is heavily conditioned by the distortion resulting from the noise inherent to signal processing.
\end{abstract}

Consequently, a complete study of the behaviour of any filtering algorithm (in the presence of noise and aperiodic components) must be carried out before its implementation.

Usually, numerical performance indices are used to measure the accuracy of a proposed method. In order to ease the analysis and comparison of different digital filters, a new set of graphic tools is presented in this paper.

To illustrate the application of these graphic tools, a case study is presented with three different filtering algorithms developed for protection relaying.

\section{Key words}

Digital filter, protection relaying, phasor estimation, signal processing, harmonic analysis.

\section{Introduction}

The correct behaviour of a protection system is essential to guarantee power quality. Nowadays, the use of devices based on power electronics technology to optimize and control the power system implies an harmonic distortion of the electrical signals. In steady-state the presence of harmonic components is not a serious problem for the phasor estimation processing. However, during transient periods the presence of a decaying dc-offset makes more difficult this harmonic analysis.

In the last years, the use of digital technologies in protection relays is increasing. Because of this, an ever growing number of filtering algorithms based on different methodologies is being developed.

A rigorous testing process must be carried out for the selected algorithm in order to cope with the practical requirements of the protection functions.

The accuracy of these algorithms is usually measured by the means of numerical indices related to the different characteristics featured by the proposed methods.

Chi-Shan Yu [1], [2] proposes the use of two indices to evaluate the filtering performance, Percentage root-meansquare error (PRMSE) and Percentage peak error (PPE) defined as follows:

$$
\begin{aligned}
\text { PRMSE } & =\frac{\sqrt{\sum_{\mathrm{k}=\mathrm{n}}^{\mathrm{n}+\mathrm{N}-1} \frac{(\text { Filter output }- \text { Steady state value })^{2}}{\mathrm{~N}}}}{\text { Steady state value }} \cdot 100 \\
\text { PPE } & =\frac{\operatorname{Max} \mid \text { Filter output }- \text { Steady state value } \mid}{\text { Steady state value }} \cdot 100
\end{aligned}
$$

Other performance indices are considered by $\mathrm{G}$. Benmouyal [3]: $\mathrm{PI}_{0}, \mathrm{PI}_{1}$ and $\mathrm{PI}_{2}$; T.S. Sidhu et al. [4]: MPD; and J. Pan et al. [5]: $\mathrm{EI}_{\mathrm{com}}$ and $\mathrm{EI}_{\mathrm{sat}}$. These indices are used to evaluate different aspects of the proposed methods.

These numerical indices provide useful information in order to compare the accuracy in the output of argument 
and module separately.

In this paper four graphics tools are proposed for integrating both outputs in a single criterion. These tools will help to a better understanding of the geometric characteristics in the filtering process.

In addition, a new numerical index is defined in order to compare the accuracy in the output of argument and module together.

\section{Graphic tools}

The four following graphic tools have been integrated in the G-CAT (Graphic - Convergence Analysis Tool) application. This application has been developed in MATLAB (MathWorks) and Mathematica (Wolfram Research) environments.

DCM (Dynamic Convergence Movie): it is an animation movie made from the respective outputs for argument and phase provided by a digital filter. Phasor oscillations lead to a quick and intuitive way to appreciate and compare the influence of aperiodic components and noise in the convergence process for different algorithms.

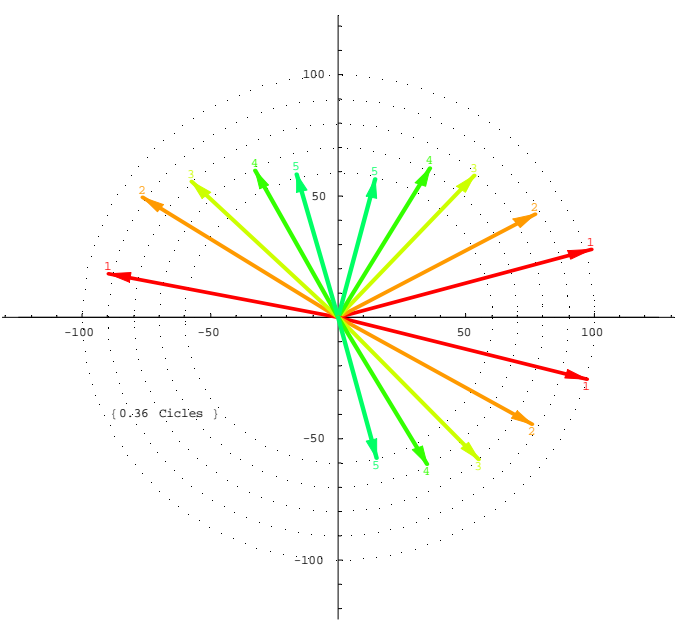

Fig. 1. Dynamic Convergence Movie (DCM) for five harmonics and three algorithms.

USD (Uncertainty Surface Diagram): it is the static representation of the information given by the corresponding DCM. This graphic is made from the successive positions of the estimated phasor during the convergence process. With a convenient colour code, comparison between the behaviour of different algorithms is very easy.

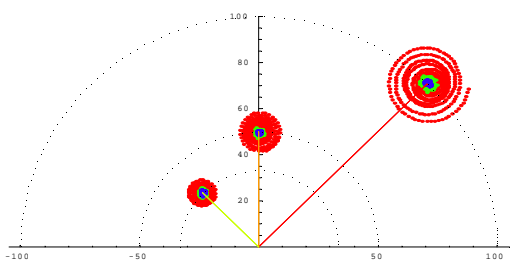

Fig. 2. Uncertainty Surface Diagram (USD) for three harmonics and three algorithms.
USDOT (Uncertainty Surface Diagram-Origin Translation): it is the USD translated to the axes origin of the plot for a specific harmonic. USDOT includes a sector diagram with the phase oscillations information.

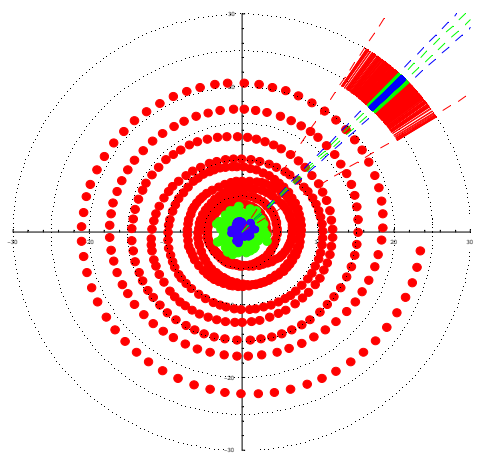

Fig. 3. Uncertainty Surface Diagram-Origin Translation (USDOT) for one harmonic and three algorithms.

USEC (Uncertainty Surface Equivalent Circle): it is the reunification in a single drawing of the phase and argument oscillations shown in the corresponding USDOT. The area of the circle is an estimation of the surface described in the DCM by the oscillating phasor. The radius gives a numerical index of the global oscillation problem when joining the separate oscillations in the phase and arguments obtained by the digital filter.

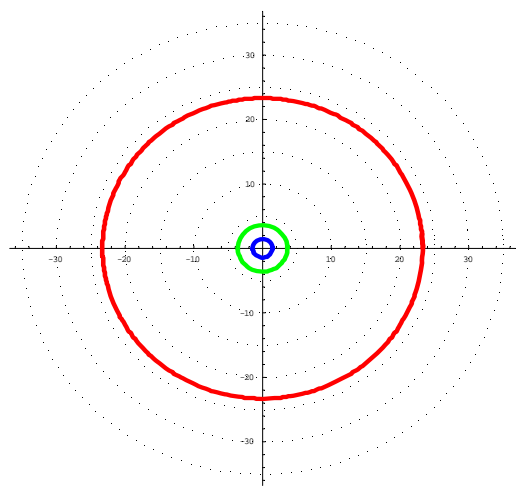

Fig. 4. Uncertainty Surface Equivalent Circle (USEC) for one harmonic and three algorithms.

The four graphic tools are intended to work in a complementary manner with the numerical indices, in order to ease the analysis and comparison of different filtering algorithms. (Figures 1 to 4 illustrate the application of the proposed tools to three digital filters and different harmonics.)

\section{Case study}

In this section, a case study is presented in which two new methods (CharmDF and s-CharmDF) [6] proposed by the authors are compared with a DFT based filtering algorithm.

The signal considered for the analysis is: 


$$
x(t)=C_{0}+C_{1} e^{-t / \tau_{1}}+C_{2} e^{-t / \tau_{2}}+\sum_{i=1}^{n} A_{i} \cos \left(\omega_{i} t+\alpha_{i}\right)
$$

where the parameters $\mathrm{C}_{0}, \mathrm{C}_{1}, \mathrm{C}_{2}, \tau_{1}, \tau_{2}, \mathrm{n}, \mathrm{A}_{\mathrm{i}}, \omega_{\mathrm{i}}$ and $\alpha_{\mathrm{i}}$ are specified in Table I.

TABLE I. - Signal parameters

\begin{tabular}{|c|c|c|c|c|c|c|c|c|}
\hline $\mathrm{C}_{0}$ & $\mathrm{C}_{1}$ & $\mathrm{C}_{2}$ & $\begin{array}{c}\tau_{1} \\
(\mathrm{~ms})\end{array}$ & $\begin{array}{c}\tau_{2} \\
(\mathrm{~ms})\end{array}$ & $\mathrm{n}$ & $\mathrm{A}_{\mathrm{i}}$ & $\begin{array}{c}\omega_{i} \\
(\mathrm{rad} / \mathrm{s})\end{array}$ & $\begin{array}{c}\alpha_{i} \\
\left({ }^{\circ}\right)\end{array}$ \\
\hline 200 & 100 & 300 & 200 & 100 & 8 & 100 & $100 \cdot \pi \cdot \mathrm{i}$ & Rand \\
\hline
\end{tabular}

Two exponential components are considered to match the case of a secondary fault current in a current transformer. Eight harmonics are taken into account as digital protections usually include an antialiasing filter to cut off the higher frequencies within the analysed signal.

The values in the signal parameters have been considered in order to test the performance of digital filter under extreme conditions.

In addition, a noise signal (in the range of $\pm 5 \%$ of $A_{1}$ ) has been superimposed to the original signal defined in (3). This will ensure the mathematical robustness of the proposed algorithms against any possible real fault conditions.

$$
y(t)=x(t)+\text { noise }(t)
$$

In the present case study, $y(t)$ has been recorded and processed with a sampling rate of 64 samples/cycle. Figure 5 shows the perturbed signal (4) and the Charm Wave corresponding to the CharmDF method applied to the perturbed signal (4). The Charm Wave is the Characteristic Harmonic Wave of $\mathrm{y}(\mathrm{t})$. This signal has complete information about the harmonic components of $y(t)$ and it eliminates the influence of aperiodic components.

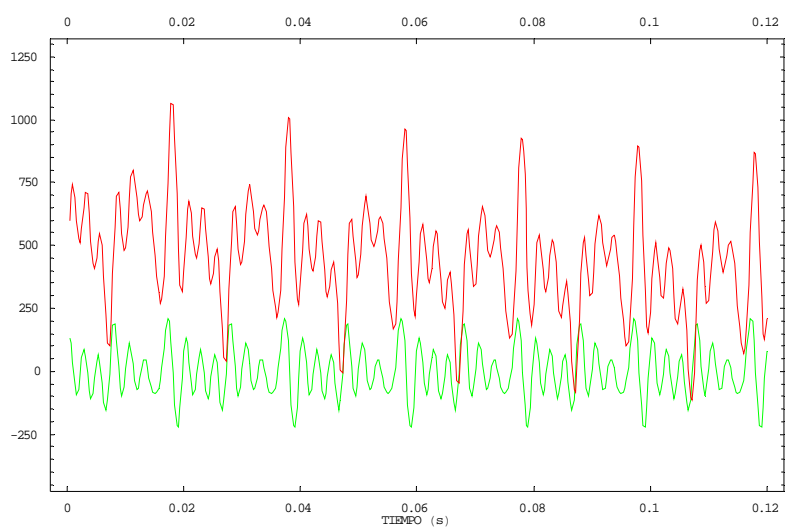

Fig. 5. Perturbed signal (red) and Charm Wave (green)

Figure 6 shows the perturbed signal (4) and the s-Charm Wave for the fundamental harmonic corresponding to the s-CharmDF method applied to the perturbed signal (4). The s-Charm Wave is the Characteristic Harmonic Wave of $\mathrm{y}(\mathrm{t})$ for a sampling slip " $\mathrm{s}$ ".

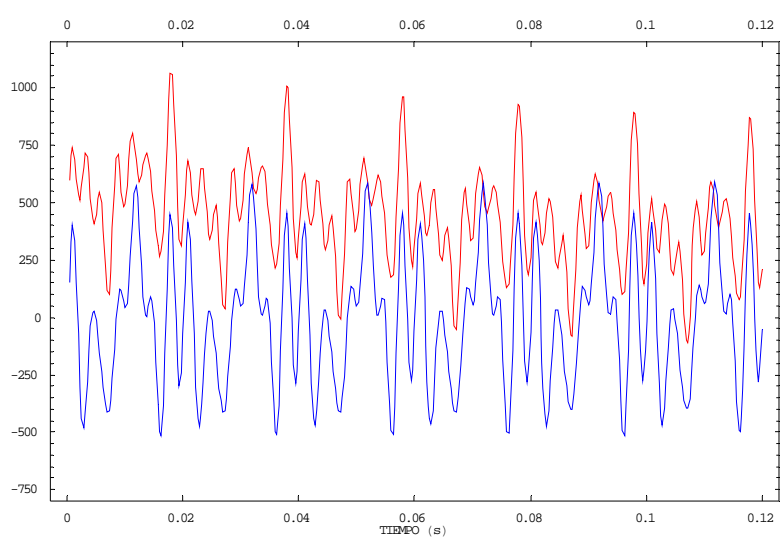

Fig. 6. Original signal (red) and s-Charm Wave (blue) for the fundamental harmonic

The outputs corresponding to the estimated module and phase of fundamental harmonic for the three aforementioned methods are shown in Figure 7 (module) and Figure 8 (phase).

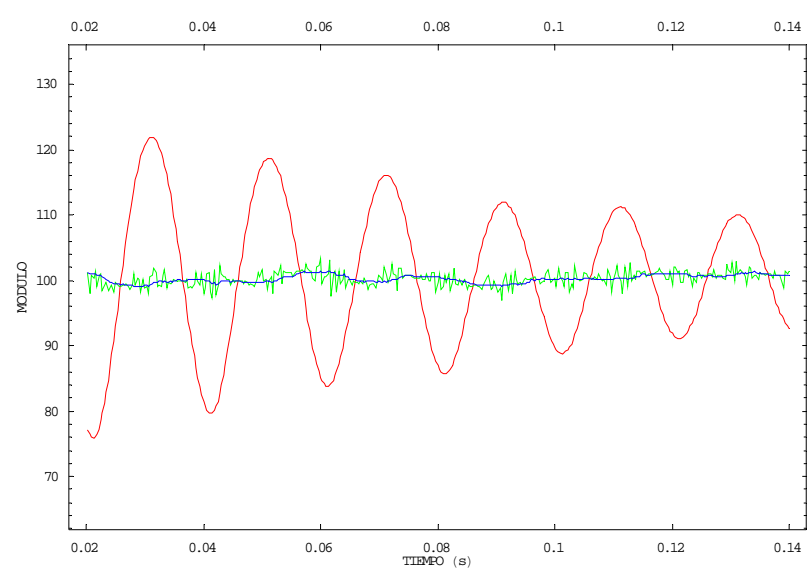

Fig. 7. Estimated module of fundamental harmonic (DFT-red, CharmDF-green and s-CharmDF-blue)

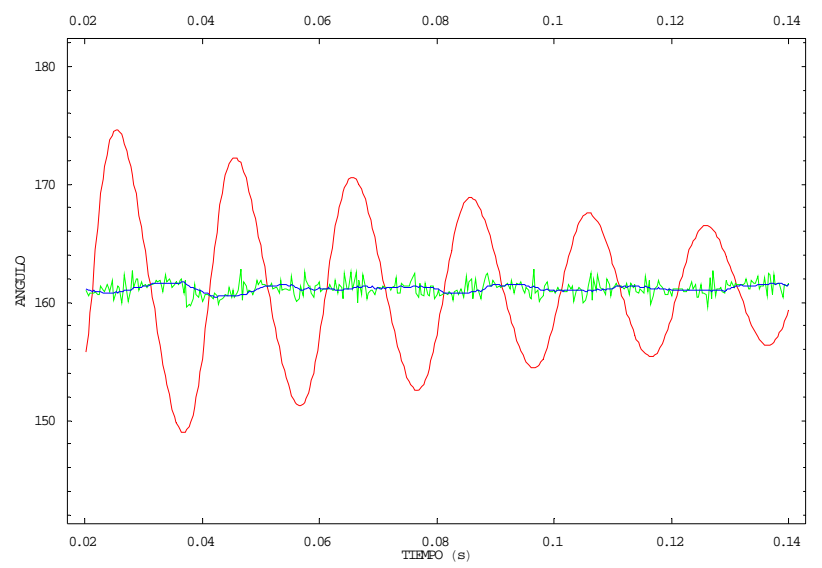

Fig. 8. Estimated phase of fundamental harmonic (DFT-red, CharmDF-green and s-CharmDF-blue)

The application of G-CAT to the present case study produces the following results for the new four graphic tools defined in Section 2. 


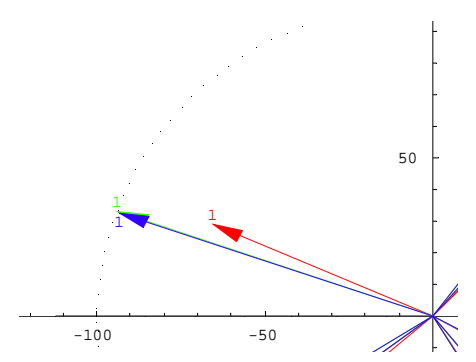

0.016 cycle

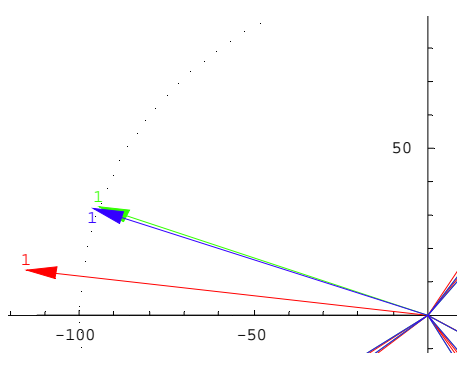

0.375 cycle

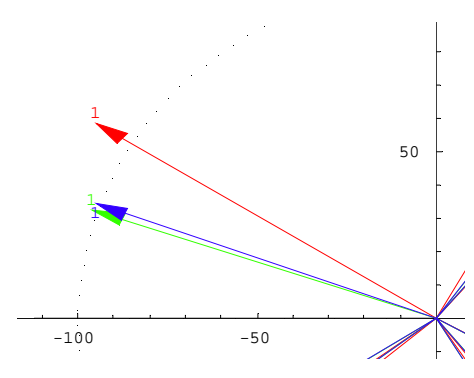

0.75 cycle

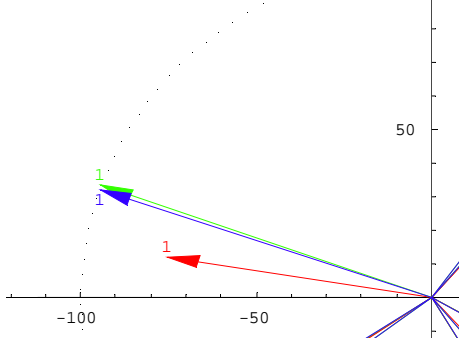

0.125 cycle

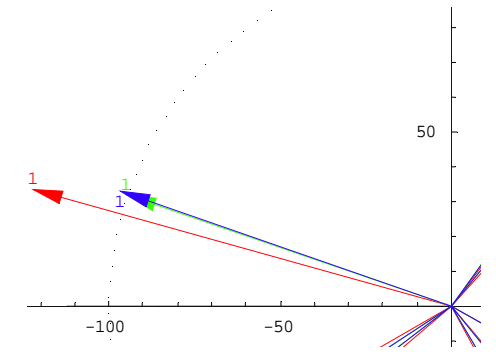

0.50 cycle

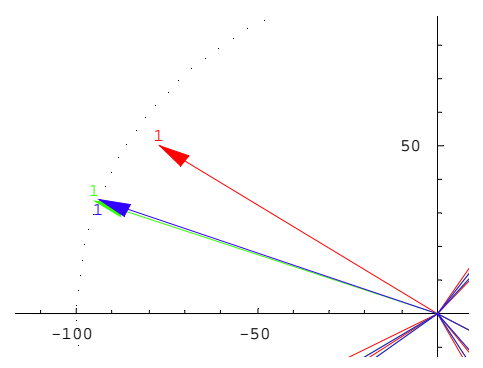

0.875 cycle

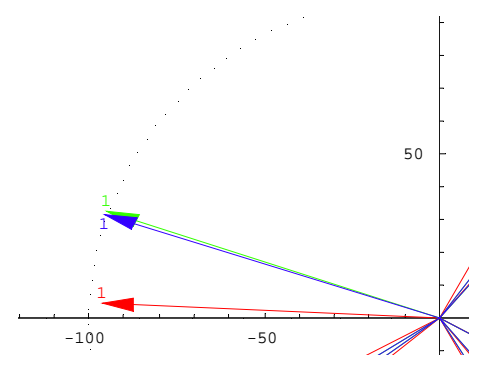

0.25 cycle

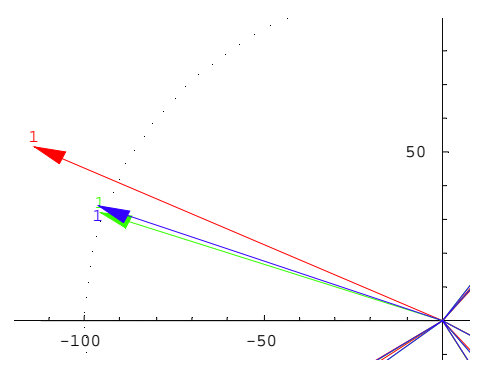

0.625 cycle

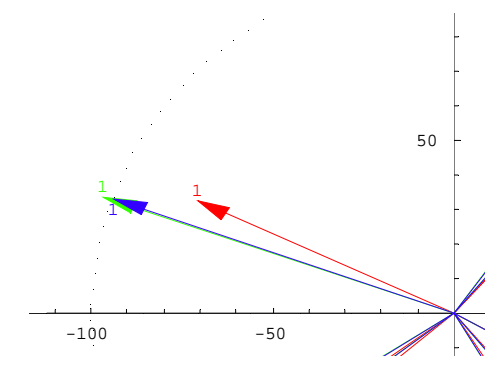

1.00 cycle

Fig. 9. DCM for the fundamental harmonic (First cycle).

The DCM for the first cycle in the estimation process of the fundamental harmonic produces the sequence of images shown in Figure 9.

The oscillation in vector output is due to influence of decaying-dc offsets and noise in the analyzed signal (4). In Figure 9 it can be seen that s-CharmDF output (blue) is more stable than CharmDF output (green) and both are much more stable than DFT output (red).

It must be remembered that DCM is a dynamic movie that can not be shown on a static platform like this paper. Therefore, Figure 9 only shows nine isolated captions corresponding to nine regularly spaced time values in the first cycle of convergence process. Obviously, the complete DCM can be run for any number of cycles.

Figure 10 shows the USD for the fundamental harmonic.

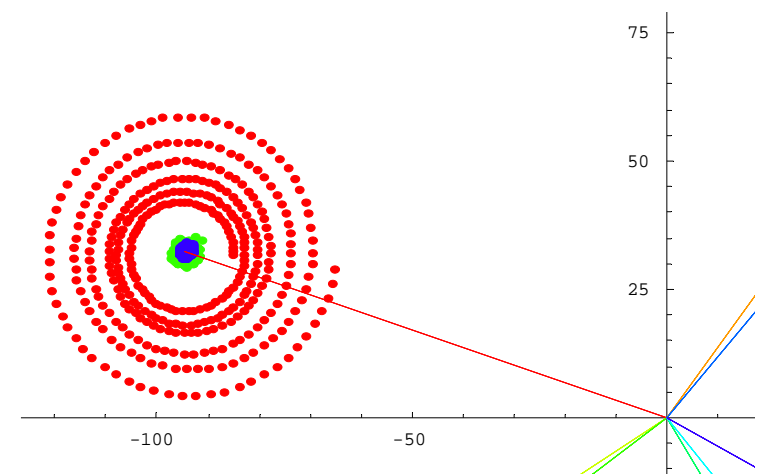

Fig. 10. USD for the fundamental harmonic (DFT-red, CharmDF-green and s-CharmDF-blue). 
The large surface corresponding to DFT is due to the oscillation (module and phase) of the vector obtained with DFT. In comparison with DFT, the less surface corresponding to CharmDF is consequence of the less oscillation of vector obtained with CharmDF. Finally, in the analysis of these three methods, Figure 10 shows clearly that s-CharmDF is the more accurate algorithm because its corresponding surface is the smallest.

The translation of the uncertainty surface diagram (Figure 10) to axes origin leads to the USDOT shown in Figure 11.

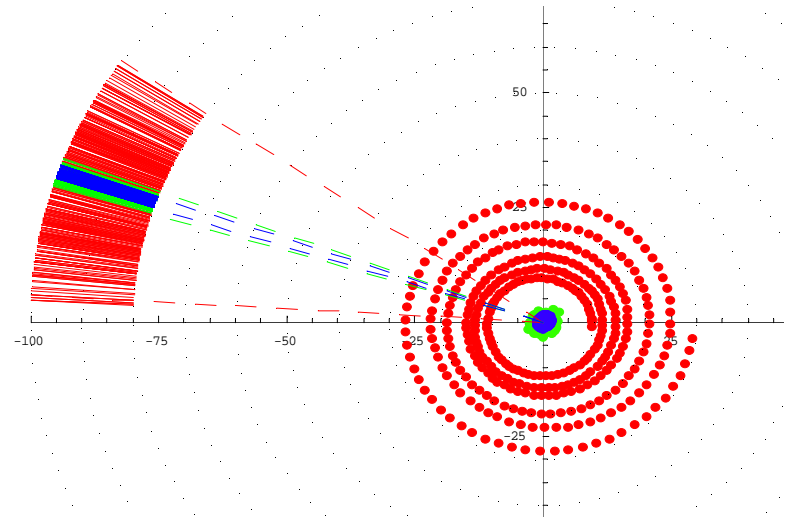

Fig. 11. USDOT for the fundamental harmonic (DFT-red, CharmDF-green and s-CharmDF-blue).

The translated plot along with the sector diagram provides a very useful insight into the geometric aspects of the convergence process.

The analysis of this USDOT leads to similar conclusions than USD analysis and it is an intermediate step in the way to the corresponding USEC (Figure 12) for the fundamental harmonic in (4).

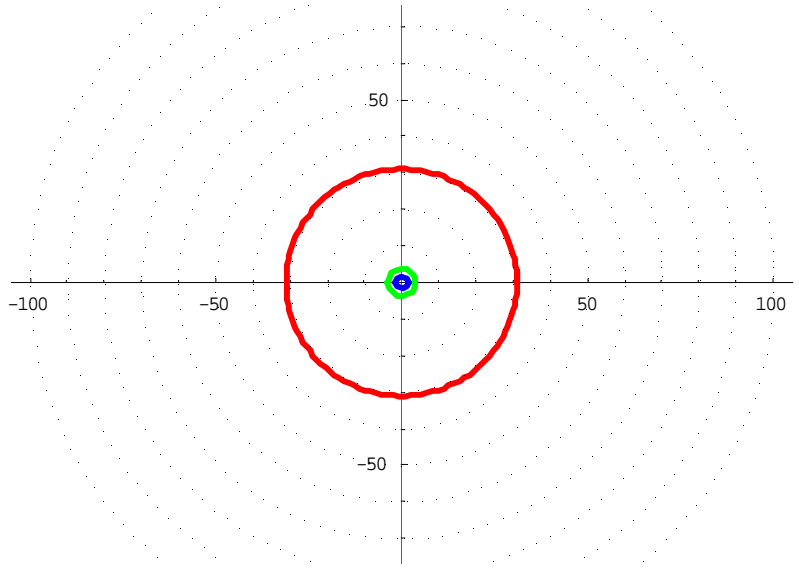

Fig. 12. USEC for the fundamental harmonic (DFT-red, CharmDF-green and s-CharmDF-blue).
The USEC area represents the joint influence of module and phase oscillations in the output of phasor estimation algorithms. Because of this, the USEC radius $\left(r_{\text {USEC }}\right)$ for each method is a numerical index of the oscillations in the convergence process. A value of $r_{\text {USEC }}=0$ corresponds with an ideal phasorial estimator totally immune to the influence of noise and aperiodic components. By the other hand, this index increases for any algorithm with its sensibility to both problems (noise and aperiodic components).

The $r_{\text {USEC }}$ values for the three methods analysed in the present case study are shown in Table II.

TABLE II. - $r_{\text {USEC }}$ values

\begin{tabular}{|c|c|c|c|}
\hline Method & DFT & CharmDF & s-CharmDF \\
\hline$r_{\text {USEC }}$ & 30.86 & 3.42 & 1.53 \\
\hline
\end{tabular}

The scale used for the aforementioned graphics tools depends on the magnitude being analysed $(\mathrm{A} / \mathrm{cm}$ or $\mathrm{V} / \mathrm{cm}$ ) and this will affect the value of $r_{\text {USEC }}$. To get rid of such influence, an adimensional ratio between $r_{\text {USEC }}$ values for two different methods may be defined. This will be of great interest in order to compare the accuracy of the two methods considered.

For example, the ratio between $r_{U S E C}$ values of DFT and CharmDF is:

$$
\frac{\left[\mathrm{r}_{\text {USEC }}\right]_{\text {DFT }}}{\left[\mathrm{r}_{\text {USEC }}\right]_{\text {CharmDF }}}=9.02
$$

Similarly, the ratio between $r_{U S E C}$ values of DFT and sCharmDF is:

$$
\frac{\left[\mathrm{r}_{\text {USEC }}\right]_{\mathrm{DFT}}}{\left[\mathrm{r}_{\text {USEC }}\right]_{\mathrm{S}-\mathrm{CharmDF}}}=20.17
$$

Finally, the ratio between $r_{U S E C}$ values of CharmDF and sCharmDF is:

$$
\frac{\left[\mathrm{r}_{\text {USEC }}\right]_{\text {CharmDF }}}{\left[\mathrm{r}_{\text {USEC }}\right]_{\text {S-CharmDF }}}=2.24
$$

From (5) and (6) is easy to conclude that both CharmDF and s-CharmDF are much more accurate than DFT. In addition, the ratio values allow to quantify the reduction in the oscillation of the convergence process. Taking into account that Charm Wave and s-Charm Wave eliminate the influence of aperiodic components, the ratio (7) shows that s-CharmDF improves the behaviour of CharmDF against noise in (4).

The results of the estimation of higher harmonics in (4) (second to eighth) are similar to the ones presented for the fundamental harmonic in this paper.

Different sets of harmonics can be taken into account with G-CAT depending on the practical application being 
considered. For example, in transformer differential protections, fundamental, second and fifth harmonics can be analysed simultaneously to evaluate the performance of the protections functions against noise and aperiodic components.

\section{Conclusions}

In this paper four graphic tools and a new numerical index have been defined in order to ease the analysis and comparison of different digital filters. These tools may be used in a great variety of ways for a deeper understanding of the convergence process.

The information provided by these graphic tools is straight forward and easily understandable for analysis purposes.

The numerical index $r_{U S E C}$ combines in a single parameter a measurement of oscillations for both phase and module in the convergence process.

The ratio of $r_{U S E C}$ values for different methods is a reliable criterion in order to compare their accuracy under different conditions.

\section{Acknowledgement}

The research work presented in this paper is a part of UPV Research Project UE05/A16, sponsored by the Basque Country University (UPV/EHU) and GE Multilin.

\section{References}

[1] C.S. Yu, “A discrete Fourier transform-based adaptive mimic phasor estimator for distance relaying applications”, IEEE Transactions on Power Delivery, Vol. 21, No. 4, pp. 1836-1846, October 2006.

[2] C.S. Yu, "A reiterative DFT to damp decaying dc and subsynchronous frequency components in fault current", IEEE Transactions on Power Delivery, Vol. 21, No. 4, pp. 1862-1870, October 2006.

[3] G. Benmouyal , "Removal of DC-offset in current waveforms using digital mimic filtering”, IEEE Transactions on Power Delivery, Vol. 10, No. 2, pp. 621-630, April 1995.

[4] T.S. Sidhu, X. Zhang, F. Albasri, M.S. Sachdev, "Discrete-Fourier-transform-based technique for removal of decaying DC offset from phasor estimates”, IEE Proc. Generation, Transmission and Distribution, Vol. 150, No. 6, pp.745-752, November 2003.

[5] J. Pan, K. Vu, Y. Hu, “An efficcient compensation algorithm for current transformer saturation effects”, IEEE Transactions on Power Delivery, Vol. 19, No. 4, pp. 1623-1628, October 2004.

[6] M.A. Zorrozua, J.F. Miñambres, J. Lázaro, I. Antiza, M. Sánchez, B. Larrea, "Estimation of Convergence Improvement introduced in the DFT by the CHARMDF (Charm Wave Digital Filter)”, X Conferencia Luso-Espanhola de Engenharia Electrotecnica, Madeira, Julio 2007. 